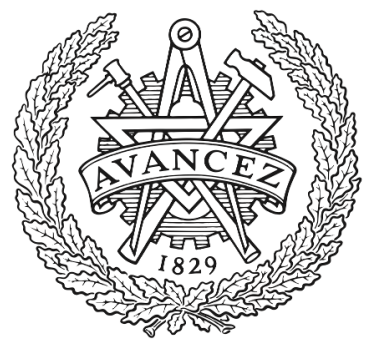

CHALMERS

UNIVERSITY OF TECHNOLOGY

\title{
Impact of Surfactants and Stabilizers on Palladium Nanoparticle-Hydrogen Interaction Kinetics: Implications for Hydrogen Sensors
}

Downloaded from: https://research.chalmers.se, 2023-04-26 14:32 UTC

Citation for the original published paper (version of record):

Stolas, A., Darmadi, I., Nugroho, F. et al (2020). Impact of Surfactants and Stabilizers on Palladium Nanoparticle-Hydrogen Interaction Kinetics:

Implications for Hydrogen Sensors. ACS Applied Nano Materials, 3(3): 2647-2653.

http://dx.doi.org/10.1021/acsanm.0c00020

N.B. When citing this work, cite the original published paper. 


\title{
Impact of Surfactants and Stabilizers on Palladium Nanoparticle- Hydrogen Interaction Kinetics: Implications for Hydrogen Sensors
}

\author{
Alicja Stolaś, Iwan Darmadi, Ferry Anggoro Ardy Nugroho, Kasper Moth-Poulsen,* \\ and Christoph Langhammer*
}

Cite This: ACS Appl. Nano Mater. 2020, 3, 2647-2653

Read Online

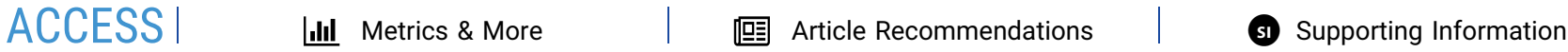

ABSTRACT: Surfactants and stabilizers are always present on the surfaces of colloidal nanocrystals due to their critical function in promoting selective facet growth and since they are essential to prevent aggregate formation in solution. After synthesis, however, the presence of these molecules on the surface of a nanocrystal is problematic because they potentially significantly alter the nature of the interaction with the environment, which is critical for sensor or catalysis applications. Here, we quantitatively scrutinize this effect experimentally for the four most common stabilizers in $\mathrm{Pd}$ nanoparticle synthesis: cetyltrimethylammonium bromide $(\mathrm{CTAB})$, tetraoctylammonium bromide (TOAB), cetyltrimethy-

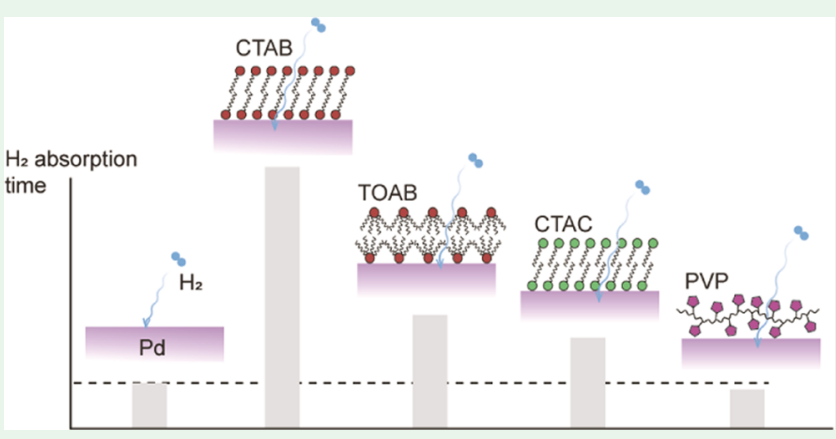
lammonium chloride (CTAC), and poly(vinylpyrrolidone) (PVP).

We use the surface-catalyzed hydrogen sorption and hydride formation reaction in $\mathrm{Pd}$ as a model system, due to its high relevance for hydrogen sensors. Specifically, we map in detail the (de)hydrogenation kinetics of arrays of nanofabricated Pd nanodisks in the presence of the surfactants and benchmark it with an uncoated Pd reference. As the key results, we find that the cationic surfactants significantly decelerate the (de)hydrogenation surface reaction, with the amplitude of deceleration mediated by the interplay between the halide-ion-Pd surface interaction strength and surfactant surface density. In contrast, a polymeric PVP coating is found to significantly accelerate hydrogen sorption. For the Pd-based hydrogen sensor application, our findings thus provide important insights for the appropriate choice of a surfactant to minimize the negative impact on hydrogen sorption kinetics and thus hydrogen detection response/recovery times. In a wider perspective, our results dramatically show how nanoparticles can attain different properties depending on what types of surfactants and stabilizers are present on their surface and how critical the quantitative understanding of their impact is for a specific application.

KEYWORDS: surfactants, palladium, hydrogen sorption, surface reaction, activation energy, nanoparticles, interactions, hydrogen sensors

\section{INTRODUCTION}

The present and potential applications of nanoparticles are ubiquitous in catalysis, energy storage, medicine, and sensing. ${ }^{1}$ In the field, there are two fundamentally different strategies to make nanoparticles and nanostructures, that is, nanofabrication and colloidal synthesis. ${ }^{2}$ Nanofabrication mainly relies on lithographic techniques, and it enables the crafting of complex nanoarchitectures in well-defined arrays with an exceptional level of control in terms of nanostructure size, shape, and arrangement on flat supports. ${ }^{2}$ On the other hand, colloidal synthesis is intrinsically more scalable, ${ }^{2}$ and the last decades have seen tremendous progress in the development of synthetic routes that allow production of nanocrystals with an excellent size and shape control. ${ }^{3}$ However, the perks of colloidal synthesis always come at the cost of nanoparticle surfaces being covered with surfactant molecules, ligands, or "stabilizers" that are necessary both to favor the growth of specific surface facets, to achieve a desired shape, and to prevent nanoparticle aggregation in solution. ${ }^{4}$ To this end, surfactant molecules have been reported to exhibit both positive and negative impacts on nanoparticle performance, depending on the application. In nanocatalysis, for example, surfactants have been found both to decrease turnover rates ${ }^{5-7}$ and to increase catalyst selectivity. ${ }^{8-11}$ In sensor applications, they have been reported to detrimentally affect response times. ${ }^{5,6}$ For these reasons, numerous attempts have been made to shed light on the role of surfactant molecules or capping ligands, particularly in the field of heterogeneous catalysis, where molecules react on the surface of nanoparticles and where surfactants thus are expected to interfere with this process. The most common way of studying surfactant effects

Received: January 3, 2020

Accepted: March 6, 2020

Published: March 6, 2020 

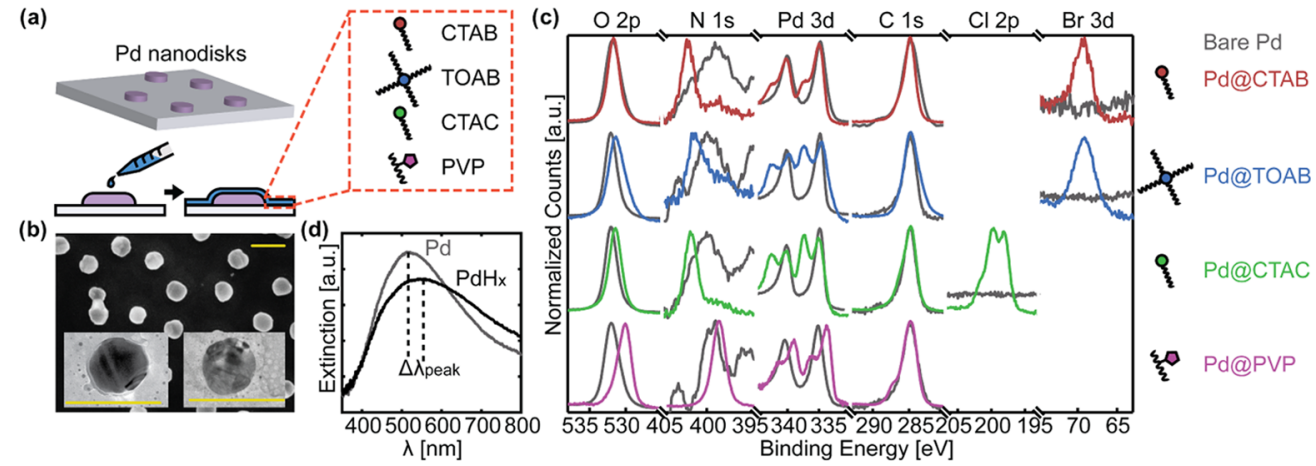

Figure 1. (a) Schematic illustration of the surfactant-coated Pd nanodisk sample preparation. The surfactant solution is dropcast onto the sample and incubated for $24 \mathrm{~h}$. Four kinds of surfactants were tested: CTAB, TOAB, CTAC, and PVP. (b) Scanning electron micrograph (SEM) of the quasirandom array of annealed Pd nanoparticles. The insets show transmission electron microscopy (TEM) images of two representative Pd nanoparticles. The scale bars correspond to $200 \mathrm{~nm}$. (c) Normalized X-ray photoelectron spectra (XPS) of relevant elements of the as-prepared Pd nanoparticles after $24 \mathrm{~h}$ annealing at $500{ }^{\circ} \mathrm{C}$ in $4 \% \mathrm{H}_{2}$ diluted in $\mathrm{Ar}$ (gray lines) and after application of the surfactant (colored lines). (d) Localized surface plasmon resonance (LSPR) as a probe for hydrogen sorption; the depicted optical extinction spectra correspond to the characteristic optical response of a Pd nanoparticle array before and after absorption of hydrogen. The spectral shift of the LSPR peak $\left(\Delta \lambda_{\text {peak }}\right)$ is utilized as readout for quantification of the response.

is to synthesize nanoparticles using different surfactants and then to compare their performance at identical conditions. ${ }^{5,6,9,12,13}$ The main problem with this approach is, however, that it may be very difficult or impossible to synthesize nanocrystals with identical size and shape (distributions) using different surfactants because fundamentally different routes may be required. ${ }^{13-15}$ This, in turn, makes it hard to unambiguously assign performance variations to different surfactant types alone. ${ }^{3,14,15}$ The second reported approach is to synthesize nanoparticles using different surfactants, to test their performance, and then to remove the surfactants either by thermal annealing or by chemical or plasma treatment, prior to a second performance test. ${ }^{16-20}$ In this way, surfactant effects are revealed by comparing nanoparticle performance before and after the cleaning step. However, also in this case, it is difficult to unambiguously assign the found differences to individual surfactant types since the studied nanocrystals synthesized using different surfactants most likely will exhibit dissimilar size and shape distributions and thus, for example, exhibit different abundances of both defects and low coordination sites. ${ }^{21}$ Furthermore, if specific cleaning procedures are applied to remove particular surfactants, they may alter the particles in various ways and thus give rise to a different response after the cleaning. ${ }^{17,18,22}$

To mitigate these uncertainties, we present here an alternative approach, which relies on the nanofabrication of surfactant-free nanoparticles, onto which a surfactant capping is applied after a baseline performance test of the surfactantfree system has been carried out. In this way, the exactly same baseline is valid for all tested ligands and the particle size and shape distributions are identical. We demonstrate our approach by nanofabricating arrays of Pd nanodisks onto flat fused silica supports. ${ }^{23}$ As a model reaction, we compare the hydrogen absorption and desorption kinetics measured using plasmonic nanospectroscopy, ${ }^{24-26}$ by quantifying both response and recovery times upon hydrogen exposure. By doing so, we are able to derive the apparent activation energies for hydride formation and decomposition in Pd covered by one of the four most commonly used ligands in colloidal Pd nanocrystal synthesis: cetyltrimethylammonium bromide ( $\mathrm{CTAB})$, tetraoctylammonium bromide (TOAB), cetyltrimethylammonium chloride (CTAC), and poly(vinylpyrrolidone)
(PVP). ${ }^{27,28}$ Such Pd nanocrystal systems have recently attracted a lot of attention both from a fundamental point of view in metal-hydrogen interactions ${ }^{29-33}$ and in applications as plasmonic hydrogen sensors. ${ }^{34}$ In the latter area, the response time is identified as one of the key challenges ${ }^{35}$ toward meeting the hydrogen sensor performance targets defined by the U.S. Department of Energy, ${ }^{36}$ and it is therefore critical to increase our understanding of the role of capping ligands in this respect. For uncoated Pd bulk and thin-film systems, the hydrogenation kinetics are well documented and thus constitute an excellent benchmark. ${ }^{37,38}$ Furthermore, to the best of our knowledge, only three related studies exist, focusing on alkyl thiols and amines. $5,6,39$

\section{RESULTS AND DISCUSSION}

Pd nanodisk arrays were nanofabricated onto optically transparent, fused silica substrates using hole-mask colloidal lithography. ${ }^{23}$ This yields large-area amorphous arrays of diskshaped nanoparticles with their diameter determined by the size of the polystyrene beads used to create the evaporation mask during nanofabrication (Figure 1a). Here, we nanofabricated $170 \mathrm{~nm}$ diameter and $25 \mathrm{~nm}$ thick Pd nanodisks. Subsequently, the particles were annealed at $500{ }^{\circ} \mathrm{C}$ in $4 \% \mathrm{H}_{2}$ diluted in $\mathrm{Ar}$ at atmospheric pressure for $24 \mathrm{~h}$ to induce recrystallization from the initially ill-defined microstructure into facetted single or polycrystalline nanoparticles with a few grains (Figure $1 \mathrm{~b}) .^{40}$

In the next step, the nanodisk surfaces were functionalized in the following different ways: (i) bare Pd control, (ii) Pd@ CTAB, (iii) Pd@TOAB, (iv) Pd@CTAC, and (v) Pd@PVP. For this purpose, the surfactants were first dissolved in deionized water and then dropcast onto the Pd nanoparticle array. Next, the excess surfactant solution was washed away in Milli-Q water, and the sample was subsequently blow-dried with $\mathrm{N}_{2}$ gas (Figures $1 \mathrm{a}$ and $\mathrm{S} 1$ ). To verify the presence of the capping ligands on the surface, we carried out XPS analysis (Figure 1c). A broad N 1s peak appears on the uncoated Pd control and can be attributed to the nitrogen contamination of the silicon substrate (see Figure S2), since nitrogen is highly unlikely to chemisorb on Pd at a low temperature. ${ }^{41,42}$ Then, sharp $\mathrm{N}$ 1s and $\mathrm{Br} 3 \mathrm{~d}$ peaks representing the cationic head of $T O A B$ and $C T A B$, respectively, are present after surfactant 
deposition, and $\mathrm{N} 1 \mathrm{~s}$ and $\mathrm{Cl} 2 \mathrm{p}$ peaks are observed for the CTAC-coated sample. For PVP, the sharp N 1s peak indicates its presence (see Tables S1 and S2 for the quantitative analysis). Furthermore, on all coated samples, the Pd $3 \mathrm{~d}$ peak exhibits a "shoulder", which is an indication for the (surface) oxidation of the Pd after surfactant deposition. This feature then disappears immediately upon exposure to $\mathrm{H}_{2}$ and the corresponding reduction of the Pd surface, as verified by XPS analysis (Figure S3).

To probe the hydrogen sorption kinetics of the five systems, we used plasmonic nanospectroscopy. ${ }^{24-26}$ It relies on the fact that the LSPR wavelength of a hydride-forming metal nanoparticle is proportional to the hydrogen uptake throughout the $\alpha$-phase region at a low-hydrogen partial pressure; the $\alpha+\beta$-phase-coexistence region ("plateau") at the first-order phase transition to and from the hydride ( $\beta$-phase); and finally, the pure $\beta$-phase region at a high-hydrogen partial pressure. Here, we used the spectral shift of the LSPR peak position $\left(\Delta \lambda_{\text {peak }}\right)$ as the readout (Figure 1d) in an experiment, where the samples were exposed to a stepwise hydrogen pressure increase/decrease to/from $320 \mathrm{mbar}$ pure $\mathrm{H}_{2}$, a pressure at which Pd hydride forms spontaneously at room temperature (at this pressure, the hydride appears as $\beta$-phase; see the pressure-composition isotherm in Figure S4). The corresponding hydrogen absorption and desorption kinetic curves are depicted in Figure 2a,b, respectively.

Under these conditions, the bare Pd control exhibits absorption and desorption times of 0.8 and $8.3 \mathrm{~s}$, respectively. The absorption and desorption times are defined as $t_{50}$ : the required time to reach $50 \%$ of the LSPR signal from the minimum (in the absorption process) or from the maximum (in the desorption process). Interestingly, all of the samples capped with cationic surfactants (i.e., CTAB, TOAB, and CTAC) exhibit significantly slower hydrogenation kinetics, but the capping does not completely prevent hydrogen absorption and desorption ( $\mathrm{Pd}$ hydride pressure-composition isotherm retained after the coating; see Figure S5). The latter observation is in agreement with Ibañez et al. who found $\mathrm{Pd} @ \mathrm{TOAB}$ nanoparticles to still respond to hydrogen, in contrast to thiolate-coated ones. ${ }^{5}$ Among the different cationic surfactants tested here, $\mathrm{CTAB}$ has the strongest effect and increases the absorption time to $4.6 \mathrm{~s}$ and desorption time to $42 \mathrm{~s}$. TOAB decelerates the kinetics to $2.0 \mathrm{~s}$ for absorption and $20 \mathrm{~s}$ for desorption. Finally, CTAC has the weakest effect and only slows absorption to $1.6 \mathrm{~s}$ and desorption to $15 \mathrm{~s}$.

To discuss the possible reasons for these observations, we remind ourselves that the conformation of cationic surfactant molecules on metallic surfaces has several characteristics that are of importance here: ${ }^{4-49}$ (i) The interaction between the surfactant and the metal surface occurs through the halide anion, whereas the hydrophobic alkyl chain points away from the metal surface. The halide anion electrostatically interacts with the cationic ammonium surfactant head. (ii) The surfactants attain a bilayer structure on the metal surface, if the concentration in solution is high enough. ${ }^{14}$ Considering these two properties, we can attribute the stabilization of the nanoparticles to a combination of electrostatic and steric interactions between the surfactants and nanoparticle surface ${ }^{50}$ (Figure S6). As the first consequence of these two factors, since the polar head groups with the hydrophobic alkyl chains interact with the Pd surface, they effectively block a significant fraction of $\mathrm{Pd}$ surface atoms. The consequently reduced number of available sites for $\mathrm{H}_{2}$ dissociation or association
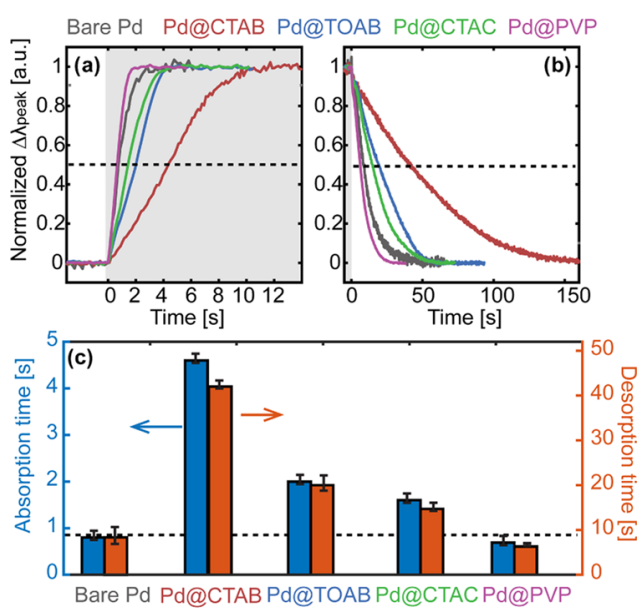

(d)

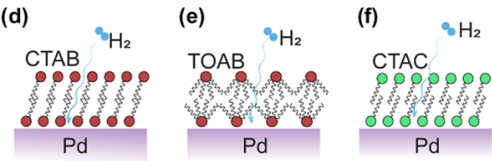

Figure 2. Bare and CTAB-, TOAB-, CTAC-, and PVP-coated Pd nanoparticle LSPR peak position shift $\left(\Delta \lambda_{\text {peak }}\right)$ (a) to stepwise exposure to $320 \mathrm{mbar} \mathrm{H}_{2}$ from vacuum and (b) to stepwise exposure to vacuum after equilibration at $320 \mathrm{mbar}_{2}$. The $\Delta \lambda_{\text {peak }}$ shift is normalized by setting $\lambda_{\text {peak }}$ of $\mathrm{Pd}$ as 0 and $\lambda_{\text {peak }}$ of saturated Pd hydride as 1 . The gray-shaded area denotes the $320 \mathrm{mbar}$ hydrogen pressure condition. The measurements were performed at $30{ }^{\circ} \mathrm{C}$. (c) Absorption and desorption times, defined as $t_{50}$, for the bare $\mathrm{Pd}$ and the four different surfactants, defined as $t_{50}$, as derived from (a) and (b). The error bars denote the standard deviation from three measurements on one sample. We note that the cationic surfactants $\mathrm{CTAB}, \mathrm{TOAB}$, and CTAC significantly decelerate the kinetics, whereas PVP accelerates both hydrogen absorption and desorption. Schematic depictions (not to scale) of the cationic surfactant adsorption on a Pd surface and the hydrogen interaction at the interface for (d) Pd@CTAB, (e) Pd@TOAB, and (f) Pd@ CTAC. $^{43-48}$

during absorption and desorption, respectively, thus increases the response time for complete (de)hydrogenation. This is in mechanistic agreement with a study of a similar system (Pt@ CTAB), where Borodko et al. observed that the polar heads reduce the accessible $\mathrm{Pt}$ surface area using $\mathrm{CO}$ as the probe molecule. ${ }^{45}$ Furthermore, it is in agreement with our CTAB adsorption coverage measurement on an annealed $\mathrm{Pd}$ film using a quartz crystal microbalance $\left(\mathrm{QCM}^{25}\right)$, which confirms a surface coverage of 1.3 molecules $/ \mathrm{nm}^{2}$ for a CTAB solution concentration of $10 \mathrm{mM}$ and assuming that the molecules form a bilayer structure (see Figure S6). This coverage is similar to the result reported by Yuan et al. ${ }^{51}$ and thus confirms the occupation of a significant fraction of surface atoms by CTAB. At the same time, it is clear that enough $\mathrm{Pd}$ sites are still available for efficient $\mathrm{H}_{2}$ dissociation even if three neighboring $\mathrm{Pd}$ atoms are required, as demonstrated for a $\operatorname{Pd}(111)$ surface, ${ }^{52,53}$ since a Pd atom on a (111) surface ${ }^{54}$ occupies $6.55 \AA^{2}$, compared to the $1.3-1.5$ molecules per $100 \AA^{2}$ for $\mathrm{CTAB}$ and CTAC, respectively.

Considering this mechanism, it is now interesting to discuss a second aspect. We find that the two Br-head surfactants $\mathrm{CTAB}$ and TOAB exhibit a significantly different slowing effect despite chemically similar polar head groups. In our mechanistic picture, this can be understood based on the fact that TOAB has four octyl chains instead of three methyl 
chains and one cetyl chain in CTAB. The projected area of $\mathrm{TOAB}$ on the surface is thus about twice as large as for CTAB in an upright packing geometry. ${ }^{55}$ Effectively, this leads to a lower $\mathrm{TOAB}$ packing density on a $\mathrm{Pd}$ surface due to steric hindrance. ${ }^{1,56}$ Moreover, the alkyl chain of TOAB is shorter than that of CTAB. A surfactant with a shorter alkyl chain has a higher degree of disorder than that of the one with a longer alkyl chain. ${ }^{57}$ Consequently, for the TOAB-capped Pd surface, more Pd sites will be available to hydrogen, which is in agreement with the observed faster response. These two different adsorption scenarios are schematically illustrated in Figure 2d (Pd@CTAB) and Figure 2e (Pd@TOAB) and agree well with a similar ligand-packing hindrance mechanism identified for catalytic surface reactions on colloidal nanoparticles in several works. ${ }^{58,59}$ Turning to the third cationic ligand studied, CTAC, it is clear that the same argument does not apply because in this case the alkyl chains are identical to CTAB. The similarity in ligand structures between CTAB and CTAC leads to a similar bilayer structure on the surface (Figure 2d vs 2f). Additionally, a corresponding QCM measurement reveals a similar adsorption coverage for $\mathrm{CTAB}$ and CTAC (1.3 vs 1.5 molecules $/ \mathrm{nm}^{2}$, respectively. See Figure S7). Nevertheless, we observe a significantly different response to hydrogen in the $\mathrm{Pd@CTAB} \mathrm{and} \mathrm{Pd@CTAC} \mathrm{systems.}$ Hence, this difference must be a consequence of the different chemistry of the anion, for which it is known that $\mathrm{Br}^{-}$interacts more strongly with $\mathrm{Pd}$ compared to $\mathrm{Cl}^{-}{ }^{46,60}$ As we discuss in detail below, this effect alters the apparent activation barrier for the rate-limiting step and thus, via a second mechanism, the hydrogen absorption and desorption times.

Now turning to the PVP system, which belongs to a different class of capping agents with only a weak interaction with the metal surface, ${ }^{61}$ we find the opposite behavior. Specifically, a PVP capping layer substantially accelerates absorption and desorption times to 0.7 and $6.3 \mathrm{~s}$, respectively (Figure 2c). This observation is in good agreement with a study by Ngene et al., who found a kinetic-enhancement effect for poly(tetrafluoroethylene) (PTFE)-coated Pd thin-film hydrogen sensors, ${ }^{62}$ and our recent conclusion that a polymer coating on Pd-based hydride-forming nanoparticles will lead to faster hydrogen absorption and desorption due to the associated reduction of the apparent activation barriers. ${ }^{63}$ At the same time, this finding is in disagreement with a recent study by Johnson et al. ${ }^{20}$ who observed a slower hydrogen uptake from colloidal Pd nanocrystals coated with PVP, which may indicate that the specific nanostructure and exposed facets may also be important or, as discussed in the introduction, this may be the consequence of the applied cleaning procedure to remove the surfactants.

As the next step of our analysis, we turn to a more quantitative discussion of the measured kinetics. Mechanistically, during hydrogen absorption, the $\mathrm{H}_{2}$ molecule first dissociates on the Pd surface, followed by the diffusion of the dissociated $\mathrm{H}^{\bullet}$ into subsurface sites as the rate-limiting step and further diffusion into interstitial lattice positions, where it occupies octahedral sites. ${ }^{6}$ Reversely, upon hydrogen desorption, the interstitial $\mathrm{H}^{\bullet}$ atoms diffuse to the surface, from which they-as the rate-limiting step-associatively desorb as $\mathrm{H}_{2}$. To extract the apparent activation energies from our experiment, we constructed Arrhenius plots for each surfactant system and the bare $\mathrm{Pd}$ control for both hydrogen absorption and desorption (Figures $3 \mathrm{a}, \mathrm{b}$ and S8 for raw data).
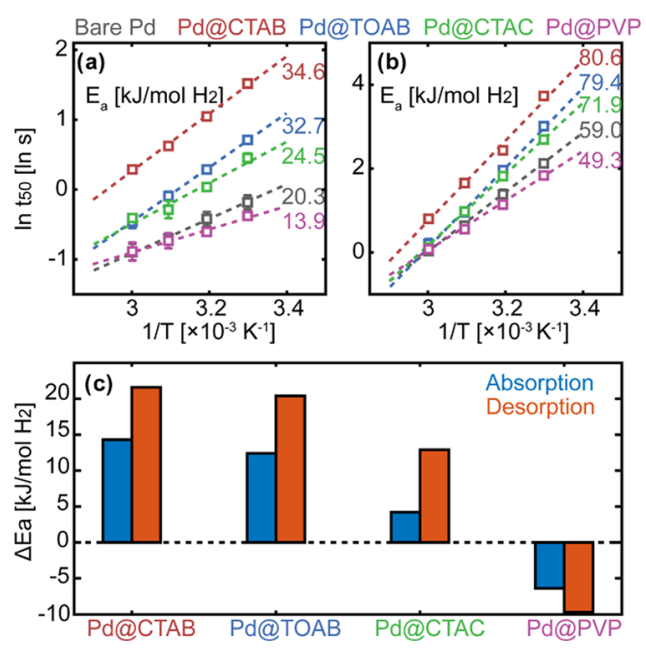

Figure 3. Arrhenius plots for $\mathrm{H}_{2}$ (a) absorption in and (b) desorption from bare and CTAB-, TOAB-, CTAC-, and PVP-coated Pd nanoparticles, respectively. $t_{50}$ is the required time to reach $50 \%$ of the optical signal from $0 \%$ (in the absorption process) and from $100 \%$ (in the desorption process). Error bars correspond to the standard deviation of three independent measurements. In most of the cases, the error bars are smaller than the symbols. (c) Apparent activation energy difference, $\Delta E_{a}$, between a bare Pd reference and the different surfactant-coated Pd samples.

We note an increase in the apparent activation energy for the cationic surfactants $\mathrm{CTAB}, \mathrm{TOAB}$, and CTAC, whereas it is reduced for the PVP stabilizer. From these Arrhenius plots, we extracted the apparent activation barriers for bare Pd, Pd@ CTAB, Pd@TOAB, Pd@CTAC, and Pd@PVP and found 20.3, 34.6, 32.7, 24.5, and $13.9 \mathrm{~kJ} / \mathrm{mol} \mathrm{H}_{2}$, respectively, for absorption and 59.0, 80.6, 79.4, 71.9, and $49.3 \mathrm{~kJ} / \mathrm{mol} \mathrm{H}_{2}$ respectively, for desorption. As evident when comparing the change in the apparent activation energy, $\Delta E_{a}$, relative to the bare $\mathrm{Pd}$ control (Figure $3 \mathrm{c}$ ), $\mathrm{CTAB}$ and $\mathrm{TOAB}$ show the largest and very similar increase in $E_{a}$, whereas $\Delta E_{\mathrm{a}}$ is significantly smaller for CTAC, corroborating that it is the strength of the interaction between the halide ion and the $\mathrm{Pd}$ surface that dictates the change in the apparent activation barrier. $^{43-45}$ In addition, the interaction between the surfactant and the Pd is obviously seen from the XPS analysis of the Pd $3 \mathrm{~d}$ peaks (Figure S9 and Table S3). Among the cationic-type surfactants, CTAB induces the largest peak position and FWHM shifts to the Pd $3 \mathrm{~d}$ peak, followed by that of TOAB and CTAC. Furthermore, this result is perfectly in line with our analysis above ( $c f$. Figure 2), where we predicted that different response times for $\mathrm{CTAB}$ and $\mathrm{TOAB}$ were mainly dictated by the different surface densities of the two systems, and not by the interaction of their cationic head groups with the Pd surface. Finally, our analysis also nicely rationalizes the found response time decrease for Pd@PVP compared to bare $\mathrm{Pd}$ as the consequence of the PVP coating reducing the apparent activation barriers, in agreement with the previous observations for other polymer coatings. ${ }^{63}$

\section{CONCLUSIONS}

We have developed an experimental approach to study the impact of the four most common surfactants and stabilizers (CTAB, TOAB, CTAC, and PVP) used in colloidal synthesis of Pd nanocrystals on their performance of catalyzing surface reactions. This approach exploits nanolithography-based 
fabrication of initially surfactant-free arrays of nanoparticles on a surface, onto which the surfactant capping is applied after having executed a baseline performance test of the surfactantfree system. As the model reaction, we compared the hydrogen absorption and desorption kinetics on Pd nanodisks by quantifying both response and recovery times upon hydrogen exposure, as well as by determining the corresponding apparent activation energies. From the experiments, we concluded that cationic surfactants $\mathrm{CTAB}, \mathrm{TOAB}$, and CTAC significantly decelerate the (de)hydrogenation surface reaction, whereas the PVP coating significantly accelerates it. For the cationic surfactants, we found the amplitude of deceleration to be dictated by the interplay between the halideion-Pd surface interaction strength and surfactant surface density. For PVP, polymer-metal bond formation lowers the hydrogen absorption and desorption apparent activation barriers and thus accelerates the kinetics. These results highlight the critical importance of quantitative understanding of the role of surfactants in a specific application of a nanocrystal system and how dramatically different properties the same type of nanoparticle can exhibit depending on the type of surfactant on its surface. Furthermore, since both positive and negative effects were observed, our findings demonstrate that the appropriate choice of a surfactant from an application perspective may significantly enhance the performance of a colloidal nanoparticle system. For the specific investigated case of $\mathrm{Pd}$-hydrogen interactions, our results are important because $\mathrm{Pd}$ nanocrystal systems have recently attracted significant attention in applications such as plasmonic hydrogen sensors. ${ }^{33}$ For such systems, the response time is identified as a key challenge ${ }^{35}$ toward meeting the hydrogen sensor performance targets defined by the U.S. Department of Energy. ${ }^{36}$ In a wider perspective, our results also point at interesting opportunities for tailoring surface catalytic properties of nanoparticles by rationally designing ligand-metal nanoparticle interactions optimized for, for instance, a specific catalytic reaction.

\section{METHODS}

Palladium Nanodisk Fabrication. Samples were fabricated on both glass (Borofloat, Schott Scandinavia $\mathrm{AB}$ ) and silicon substrates with hole-mask colloidal lithography (following procedures detailed elsewhere $\left.{ }^{64,65}\right)$. The Pd evaporation pellet was provided by Kurt J. Lesker Company (purity 99.95\%). The average diameter of Pd nanodisks is $170 \mathrm{~nm}$, dictated by the average size of colloidal polystyrene nanobeads used to create the mask during nanofabrication. The height of the nanodisks is $25 \mathrm{~nm}$. Subsequent thermal annealing at $500{ }^{\circ} \mathrm{C}$ in $4 \% \mathrm{H}_{2}$ in $\mathrm{Ar}$ carrier gas for $24 \mathrm{~h}$ leads to recrystallization and $\mathrm{Pd}$ nanodisk diameter shrinkage of a few nanometers.

Surfactant Coating Procedures. The surfactants CTAB (SigmaAldrich, purity $\geq 98 \%$ ), TOAB (Sigma-Aldrich, purity 98\%), CTAC (TCI, purity $>95.0 \%$ ), or PVP (Sigma-Aldrich, $M_{\mathrm{w}} \sim 55000$ ) dissolved at 1 or $10 \mathrm{mM}$ concentrations in Milli-Q water were dropcast onto the sample surface. For that purpose, the sample was placed on a three-dimensional (3D)-printed scaffold, which after dropcasting was transferred to a Petri dish with an attached lid to incubate for $24 \mathrm{~h}$. Subsequently, the excess surfactant solution was washed away in Milli-Q water and the sample was blow-dried with $\mathrm{N}_{2}$ gas.

Transmission Electron Microscopy (TEM) and Scanning Electron Microscopy (SEM). Pd nanodisks before and after annealing, nanofabricated onto a $\mathrm{SiN}_{x}$ membrane, were imaged using a Tecnai T20 TEM microscope, operating at $200 \mathrm{kV}$ with a LaB6 gun. For SEM imaging, a Zeiss Supra $60 \mathrm{VP}$ was used. The samples were prepared on a silicon wafer to minimize charging during imaging. The electron beam energy was $10 \mathrm{kV}$. The working distance was $5 \mathrm{~mm}$, and an in-lens detector was employed.

Hydrogenation Kinetics Measurements. The kinetics measurements were performed in a custom-made vacuum chamber setup with optical windows reported earlier. ${ }^{65}$ The absolute hydrogen pressure in the chamber was monitored using two capacitive pressure gauges with different pressure ranges (MKS Baratron). Optical transmittance measurements through the sample were enabled by UHV-compatible sapphire windows mounted on the vacuum chamber and by using a fiber-coupled, unpolarized white light source (Avantes AvaLight-Hal) and a fixed-grating, fiber-coupled spectrophotometer (Avantes SensLine AvaSpec-2048XL). The pressure inside the chamber was controlled using a microbar-precision leak valve. The temperature was controlled with a heating coil wrapped around the chamber and a temperature controller (Eurotherm 3216N) in a feedback loop manner, where the sample surface temperature inside the vacuum chamber was continuously used as the input. The LSPR peak descriptor, $\lambda_{\text {peak }}$, was obtained by fitting a Lorentzian function to the LSPR peak in the measured optical extinction spectra.

X-ray Photoelectron Spectroscopy (XPS). A PerkinElmer PHI $5000 \mathrm{C}$ XPS system was used, and the samples were prepared on a silicon substrate to minimize charge buildup. The scanning resolution was $0.125 \mathrm{eV}$, and the $\mathrm{C}-\mathrm{C} / \mathrm{C}-\mathrm{H} 1$ s peak was set to $384.5 \mathrm{eV}$ as reference. For analysis, Multipak 6.0 software was employed with a Shirley-type background correction.

Quartz Crystal Microbalance (QCM). The QCM measurements were performed to measure the surfactant adsorption coverage on a Pd film (Q-Sense AB). A $100 \mathrm{~nm}$ Pd film was evaporated by e-beam PVD onto a Q-Sense QSX 303 sensor and annealed at $450{ }^{\circ} \mathrm{C}$ for 24 $\mathrm{h}$ to induce crystallization. CTAB and CTAC solutions $(10 \mathrm{mM})$ diluted in Milli-Q water were prepared. The QCM experiment was carried out at a constant flow rate of $50 \mu \mathrm{L} / \mathrm{min}$, and the module temperature was kept at $35{ }^{\circ} \mathrm{C}$. The adsorption measurement procedure consists of Milli- $\mathrm{Q}$ water flush (as baseline) followed by a surfactant solution flush. It is worth noting that a corresponding TOAB measurement was not possible due to clogging of the QCM flow system.

\section{ASSOCIATED CONTENT}

\section{Supporting Information}

The Supporting Information is available free of charge at https://pubs.acs.org/doi/10.1021/acsanm.0c00020.

Surfactant/stabilizer deposition procedure, XPS spectra of surfactant-coated palladium after a hydrogen flush, quantitative XPS elemental analysis of surfactant-coated palladium, schematic illustration of a surfactant-Pd nanoparticle stabilization, Pd 3d peak XPS analysis, $\mathrm{CTAB}$ and CTAC adsorption coverage measurements by QCM, (coated/uncoated) palladium pressure-peak position isotherm, and raw data of the Arrhenius plot (PDF)

\section{AUTHOR INFORMATION}

\section{Corresponding Authors}

Kasper Moth-Poulsen - Department of Chemistry and Chemical Engineering, Chalmers University of Technology, 412 96 Göteborg, Sweden; 이이이.org/0000-0003-4018-4927; Email: kasper.moth-poulsen@chalmers.se

Christoph Langhammer - Department of Physics, Chalmers University of Technology, 41296 Göteborg, Sweden; ○ orcid.org/0000-0003-2180-1379; Email: clangham@ chalmers.se 


\section{Authors}

Alicja Stolas - Department of Chemistry and Chemical Engineering, Chalmers University of Technology, 41296 Göteborg, Sweden; 이이이.org/0000-0002-6736-9553

Iwan Darmadi - Department of Physics, Chalmers University of Technology, 41296 Göteborg, Sweden; (i) orcid.org/00000002-5921-9336

Ferry Anggoro Ardy Nugroho - Department of Physics, Chalmers University of Technology, 41296 Göteborg, Sweden; (1) orcid.org/0000-0001-5571-0454

Complete contact information is available at:

https://pubs.acs.org/10.1021/acsanm.0c00020

\section{Author Contributions}

A.S. and I.D. contributed equally. The manuscript was written through contributions of all authors. All authors have given approval to the final version of the manuscript.

\section{Notes}

The authors declare no competing financial interest.

\section{ACKNOWLEDGMENTS}

We acknowledge financial support from the Swedish Foundation for Strategic Research framework project RMA15-0052. Part of this work was carried out at the Chalmers Cleanroom Facility and Chalmers Materials Analysis Laboratory. We also thank Dr. Johnas Eklöf-Österberg for drawing two figures for this paper.

\section{REFERENCES}

(1) Heinz, H.; Pramanik, C.; Heinz, O.; Ding, Y.; Mishra, R. K.; Marchon, D.; Flatt, R. J.; Estrela-Lopis, I.; Llop, J.; Moya, S.; Ziolo, R. F. Nanoparticle Decoration with Surfactants: Molecular Interactions, Assembly, and Applications. Surf. Sci. Rep. 2017, 72, 1-58.

(2) Zhang, Q.; Tan, Y. N.; Xie, J.; Lee, J. Y. Colloidal Synthesis of Plasmonic Metallic Nanoparticles. Plasmonics 2009, 4, 9-22.

(3) An, K.; Somorjai, G. A. Size and Shape Control of Metal Nanoparticles for Reaction Selectivity in Catalysis. ChemCatChem 2012, 4, 1512-1524.

(4) Merg, A. D.; Zhou, Y.; Smith, A. M.; Millstone, J. E.; Rosi, N. L. Ligand Exchange for Controlling the Surface Chemistry and Properties of Nanoparticle Superstructures. ChemNanoMat 2017, 3, $745-749$.

(5) Ibañez, F. J.; Zamborini, F. P. Reactivity of Hydrogen with SolidState Films of Alkylamine- and Tetraoctylammonium BromideStabilized Pd, PdAg, and PdAu Nanoparticles for Sensing and Catalysis Applications. J. Am. Chem. Soc. 2008, 130, 622-633.

(6) Moreno, M.; Ibañez, F. J.; Jasinski, J. B.; Zamborini, F. P. Hydrogen Reactivity of Palladium Nanoparticles Coated with Mixed Monolayers of Alkyl Thiols and Alkyl Amines for Sensing and Catalysis Applications. J. Am. Chem. Soc. 2011, 133, 4389-4397.

(7) Samiey, B.; Cheng, C.-H.; Wu, J. Effects of Surfactants on the Rate of Chemical Reactions. J. Chem. 2014, 2014, 1-14.

(8) Marshall, S. T.; O’Brien, M.; Oetter, B.; Corpuz, A.; Richards, R. M.; Schwartz, D. K.; Medlin, J. W. Controlled Selectivity for Palladium Catalysts Using Self-Assembled Monolayers. Nat. Mater. 2010, 9, 853-858.

(9) Kwon, S. G.; Krylova, G.; Sumer, A.; Schwartz, M. M.; Bunel, E. E.; Marshall, C. L.; Chattopadhyay, S.; Lee, B.; Jellinek, J.; Shevchenko, E. V. Capping Ligands as Selectivity Switchers in Hydrogenation Reactions. Nano Lett. 2012, 12, 5382-5388.

(10) Almora-Barrios, N.; Vilé, G.; Garcia-Ratés, M.; Pérez-Ramírez, J.; López, N. Electrochemical Effects at Surfactant-Platinum Nanoparticle Interfaces Boost Catalytic Performance. ChemCatChem 2017, 9, 604-609.
(11) Zhao, Y.; Baeza, J. A.; Koteswara Rao, N.; Calvo, L.; Gilarranz, M. A.; Li, Y. D.; Lefferts, L. Unsupported PVA- and PVP-Stabilized Pd Nanoparticles as Catalyst for Nitrite Hydrogenation in Aqueous Phase. J. Catal. 2014, 318, 162-169.

(12) Jones, S.; Qu, J.; Tedsree, K.; Gong, X.-Q.; Tsang, S. C. E. Prominent Electronic and Geometric Modifications of Palladium Nanoparticles by Polymer Stabilizers for Hydrogen Production under Ambient Conditions. Angew. Chem., Int. Ed. 2012, 51, 11275-11278.

(13) Ma, L.; Sinyukov, A. M.; Chen, Y.; Kundu, S.; Liang, H.; Dai, W.; Yi, S.-I. Morphology Dependent Catalysis and Surface Enhanced Raman Scattering (SERS) Studies Using Pd Nanostructures in DNA, CTAB and PVA Scaffolds. Dalton Trans. 2017, 46, 9678-9691.

(14) Kittler, S.; Hickey, S. G.; Wolff, T.; Eychmüller, A. Easy and Fast Phase Transfer of CTAB Stabilised Gold Nanoparticles from Water to Organic Phase. Z. Physiol. Chem. 2015, 229, 235-245.

(15) Dewi, M. R.; Laufersky, G.; Nann, T. A Highly Efficient Ligand Exchange Reaction on Gold Nanoparticles: Preserving Their Size, Shape and Colloidal Stability. RSC Adv. 2014, 4, 34217-34220.

(16) Jana, D.; Matti, C.; He, J.; Sagle, L. Capping Agent-Free Gold Nanostars Show Greatly Increased Versatility and Sensitivity for Biosensing. Anal. Chem. 2015, 87, 3964-3972.

(17) Naresh, N.; Wasim, F. G. S.; Ladewig, B. P.; Neergat, M. Removal of Surfactant and Capping Agent from Pd Nanocubes (PdNCs) Using Tert-Butylamine: Its Effect on Electrochemical Characteristics. J. Mater. Chem. A 2013, 1, 8553.

(18) Wu, J.; Zeng, L.; Cheng, D.; Chen, F.; Zhan, X.; Gong, J. Synthesis of Pd Nanoparticles Supported on $\mathrm{CeO} 2$ Nanotubes for CO Oxidation at Low Temperatures. Chinese J. Catal. 2016, 37, 8390.

(19) Collins, G.; Davitt, F.; O’Dwyer, C.; Holmes, J. D. Comparing Thermal and Chemical Removal of Nanoparticle Stabilizing Ligands: Effect on Catalytic Activity and Stability. ACS Appl. Nano Mater 2018, 1, 7129-7138.

(20) Johnson, N. J. J.; Lam, B.; Sherbo, R. S.; Fork, D. K.; Berlinguette, C. P. Ligands Affect Hydrogen Absorption and Desorption by Palladium Nanoparticles. Chem. Mater. 2019, 31, 8679-8684.

(21) Jung, S. C.; Park, Y.-K.; Jung, H.-Y.; Kim, S. C. Effect of Stabilizing Agents on the Synthesis of Palladium Nanoparticles. J. Nanosci. Nanotechnol. 2017, 17, 2833-2836.

(22) Li, D.; Wang, C.; Tripkovic, D.; Sun, S.; Markovic, N. M.; Stamenkovic, V. R. Surfactant Removal for Colloidal Nanoparticles from Solution Synthesis: The Effect on Catalytic Performance. ACS Catal. 2012, 2, 1358-1362.

(23) Fredriksson, H.; Alaverdyan, Y.; Dmitriev, A.; Langhammer, C.; Sutherland, D. S.; Zäch, M.; Kasemo, B. Hole-Mask Colloidal Lithography. Adv. Mater. 2007, 19, 4297-4302.

(24) Langhammer, C.; Zorić, I.; Kasemo, B.; Clemens, B. M. Hydrogen Storage in Pd Nanodisks Characterized with a Novel Nanoplasmonic Sensing Scheme. Nano Lett. 2007, 7, 3122-3127.

(25) Nugroho, F. A. A.; Darmadi, I.; Zhdanov, V. P.; Langhammer, C. Universal Scaling and Design Rules of Hydrogen-Induced Optical Properties in Pd and Pd-Alloy Nanoparticles. ACS Nano 2018, 12, 9903-9912.

(26) Poyli, M. A.; Silkin, V. M.; Chernov, I. P.; Echenique, P. M.; Muiño, R. D.; Aizpurua, J. Multiscale Theoretical Modeling of Plasmonic Sensing of Hydrogen Uptake in Palladium Nanodisks. J. Phys. Chem. Lett. 2012, 3, 2556-2561.

(27) Cookson, J. The Preparation of Palladium Nanoparticles. Platin. Met. Rev. 2012, 56, 83-98.

(28) Li, C.; Sato, T.; Yamauchi, Y. Size-Controlled Synthesis of Mesoporous Palladium Nanoparticles as Highly Active and Stable Electrocatalysts. Chem. Commun. 2014, 50, 11753-11756.

(29) Syrenova, S.; Wadell, C.; Nugroho, F. A. A.; Gschneidtner, T. A.; Diaz Fernandez, Y. A.; Nalin, G.; Switlik, D.; Westerlund, F.; Antosiewicz, T. J.; Zhdanov, V. P.; Moth-Poulsen, K.; Langhammer, C. Hydride Formation Thermodynamics and Hysteresis in Individual Pd Nanocrystals with Different Size and Shape. Nat. Mater. 2015, 14, $1236-1244$. 
(30) Griessen, R.; Strohfeldt, N.; Giessen, H. Thermodynamics of the Hybrid Interaction of Hydrogen with Palladium Nanoparticles. Nat. Mater. 2016, 15, 311-317.

(31) Narayan, T. C.; Hayee, F.; Baldi, A.; Leen Koh, A.; Sinclair, R.; Dionne, J. A. Direct Visualization of Hydrogen Absorption Dynamics in Individual Palladium Nanoparticles. Nat. Commun. 2017, 8, No. 14020.

(32) Baldi, A.; Narayan, T. C.; Koh, A. L.; Dionne, J. A. In Situ Detection of Hydrogen-Induced Phase Transitions in Individual Palladium Nanocrystals. Nat. Mater. 2014, 13, 1143-1148.

(33) Hayee, F.; Narayan, T. C.; Nadkarni, N.; Baldi, A.; Koh, A. L.; Bazant, M. Z.; Sinclair, R.; Dionne, J. A. In-Situ Visualization of Solute-Driven Phase Coexistence within Individual Nanorods. Nat. Commun. 2018, 9, No. 1775.

(34) Wadell, C.; Syrenova, S.; Langhammer, C. Plasmonic Hydrogen Sensing with Nanostructured Metal Hydrides. ACS Nano 2014, 8, 11925-11940.

(35) Penner, R. M. A Nose for Hydrogen Gas: Fast, Sensitive H2 Sensors Using Electrodeposited Nanomaterials. Acc. Chem. Res. 2017, 50, 1902-1910.

(36) Fuel Cell Technologies Office Multi-Year Research, Development, and Demonstration Plan, 3.7 Hydrogen Safety, Codes, and Standards; U.S. Department of Energy, 2015; p 21.

(37) Auer, W.; Grabke, H. J. The Kinetics of Hydrogen Absorption in Palladium ( $\alpha$ - and $\beta$-Phase) and Palladium-Silver-Alloys. Ber. Bunsen-Ges. Phys. Chem. 1974, 78, 58-67.

(38) Kay, B. D.; Peden, C. H. F.; Goodman, D. W. Kinetics of Hydrogen Absorption by Pd(110). Phys. Rev. B 1986, 34, 817-822.

(39) Ibañez, F. J.; Zamborini, F. P. Ozone- and Thermally Activated Films of Palladium Monolayer-Protected Clusters for Chemiresistive Hydrogen Sensing. Langmuir 2006, 22, 9789-9796.

(40) Alekseeva, S.; da Silva Fanta, A. B.; Iandolo, B.; Antosiewicz, T. J.; Nugroho, F. A. A.; Wagner, J. B.; Burrows, A.; Zhdanov, V. P.; Langhammer, C. Grain Boundary Mediated Hydriding Phase Transformations in Individual Polycrystalline Metal Nanoparticles. Nat. Commun. 2017, 8, No. 1084.

(41) Miyazaki, E. Chemisorption of Diatomic Molecules (H2, N2, CO) on Transition d-Metals. J. Catal. 1980, 65, 84-94.

(42) Miyazaki, E.; Kojima, I.; Kojima, S. Chemisorption of Molecular Nitrogen on Palladium Surfaces at and above Room Temperature. Langmuir 1985, 1, 264-266.

(43) Nikoobakht, B.; El-Sayed, M. A. Evidence for Bilayer Assembly of Cationic Surfactants on the Surface of Gold Nanorods. Langmuir 2001, 17, 6368-6374.

(44) Sui, Z. M.; Chen, X.; Wang, L. Y.; Xu, L. M.; Zhuang, W. C.; Chai, Y. C.; Yang, C. J. Capping Effect of CTAB on Positively Charged Ag Nanoparticles. Phys. E 2006, 33, 308-314.

(45) Borodko, Y.; Jones, L.; Lee, H.; Frei, H.; Somorjai, G. Spectroscopic Study of Tetradecyltrimethylammonium Bromide PtC14TAB Nanoparticles: Structure and Stability. Langmuir 2009, 25, 6665-6671.

(46) Lohse, S. E.; Burrows, N. D.; Scarabelli, L.; Liz-Marzán, L. M.; Murphy, C. J. Anisotropic Noble Metal Nanocrystal Growth: The Role of Halides. Chem. Mater. 2014, 26, 34-43.

(47) Lohse, S. E.; Murphy, C. J. The Quest for Shape Control: A History of Gold Nanorod Synthesis. Chem. Mater. 2013, 25, 12501261.

(48) Langille, M. R.; Personick, M. L.; Zhang, J.; Mirkin, C. A. Defining Rules for the Shape Evolution of Gold Nanoparticles. J. Am. Chem. Soc. 2012, 134, 14542-14554.

(49) Jain, T.; Tehrani-Bagha, A. R.; Shekhar, H.; Crawford, R.; Johnson, E.; Nørgaard, K.; Holmberg, K.; Erhart, P.; Moth-Poulsen, K. Anisotropic Growth of Gold Nanoparticles Using Cationic Gemini Surfactants: Effects of Structure Variations in Head and Tail Groups. J. Mater. Chem. C 2014, 2, 994-1003.

(50) Cookson, J. The Preparation of Palladium Nanoparticles. Platin. Met. Rev. 2012, 56, 83-98.

(51) Yuan, S.; Ma, L.; Zhang, X.; Zheng, L. Molecular Dynamics Studies on Monolayer of Cetyltrimethylammonium Bromide
Surfactant Formed at the Air/Water Interface. Colloids Surf., A 2006, 289, 1-9.

(52) Lopez, N.; Łodziana, Z.; Illas, F.; Salmeron, M. When Langmuir Is Too Simple: $\mathrm{H} 2$ Dissociation on $\mathrm{Pd}(111)$ at High Coverage. Phys. Rev. Lett. 2004, 93, No. 146103.

(53) Mitsui, T.; Rose, M. K.; Fomin, E.; Ogletree, D. F.; Salmeron, M. Dissociative Hydrogen Adsorption on Palladium Requires Aggregates of Three or More Vacancies. Nature 2003, 422, 705-707.

(54) Paul, J.; Sautet, P. Density-Functional Periodic Study of the Adsorption of Hydrogen on a Palladium (111) Surface. Phys. Rev. B 1996, 53, 8015-8027.

(55) de Barros, H. R.; Piovan, L.; Sassaki, G. L.; de Araujo Sabry, D.; Mattoso, N.; Nunes, Á. M.; Meneghetti, M. R.; Riegel-Vidotti, I. C. Surface Interactions of Gold Nanorods and Polysaccharides: From Clusters to Individual Nanoparticles. Carbohydr. Polym. 2016, 152, $479-486$

(56) Heinz, H.; Vaia, R. A.; Farmed, B. L. Relation between Packing Density and Thermal Transitions of Alkyl Chains on Layered Silicate and Metal Surfaces. Langmuir 2008, 24, 3727-3733.

(57) Wang, J.; Han, B.; Dai, M.; Yan, H.; Li, Z.; Thomas, R. K. Effects of Chain Length and Structure of Cationic Surfactants on the Adsorption onto Na-Kaolinite. J. Colloid Interface Sci. 1999, 213, 596601.

(58) Albani, D.; Vilé, G.; Mitchell, S.; Witte, P. T.; Almora-Barrios, N.; Verel, R.; López, N.; Pérez-Ramírez, J. Ligand Ordering Determines the Catalytic Response of Hybrid Palladium Nanoparticles in Hydrogenation. Catal. Sci. Technol. 2016, 6, 1621-1631.

(59) Vilé, G.; Almora-Barrios, N.; Mitchell, S.; Lõpez, N.; PérezRamírez, J. From the Lindlar Catalyst to Supported Ligand-Modified Palladium Nanoparticles: Selectivity Patterns and Accessibility Constraints in the Continuous-Flow Three-Phase Hydrogenation of Acetylenic Compounds. Chem. Eur. J. 2014, 20, 5926-5937.

(60) Meena, S. K.; Celiksoy, S.; Schäfer, P.; Henkel, A.; Sönnichsen, C.; Sulpizi, M. The Role of Halide Ions in the Anisotropic Growth of Gold Nanoparticles: A Microscopic, Atomistic Perspective. Phys. Chem. Chem. Phys. 2016, 18, 13246-13254.

(61) Koczkur, K. M.; Mourdikoudis, S.; Polavarapu, L.; Skrabalak, S. E. Polyvinylpyrrolidone (PVP) in Nanoparticle Synthesis. Dalton Trans. 2015, 44, 17883-17905.

(62) Ngene, P.; Westerwaal, R. J.; Sachdeva, S.; Haije, W.; de Smet, L. C. P. M.; Dam, B. Polymer-Induced Surface Modifications of PdBased Thin Films Leading to Improved Kinetics in Hydrogen Sensing and Energy Storage Applications. Angew. Chem., Int. Ed. 2014, 53, 12081-12085.

(63) Nugroho, F. A. A.; Darmadi, I.; Cusinato, L.; Susarrey-Arce, A.; Schreuders, H.; Bannenberg, L. J.; da Silva Fanta, A. B.; Kadkhodazadeh, S.; Wagner, J. B.; Antosiewicz, T. J.; Hellman, A.; Zhdanov, V. P.; Dam, B.; Langhammer, C. Metal-Polymer Hybrid Nanomaterials for Plasmonic Ultrafast Hydrogen Detection. Nat. Mater. 2019, 18, 489-495.

(64) Nugroho, F. A. A.; Iandolo, B.; Wagner, J. B.; Langhammer, C. Bottom-Up Nanofabrication of Supported Noble Metal Alloy Nanoparticle Arrays for Plasmonics. ACS Nano 2016, 10, 2871-2879.

(65) Wagner, J. B.; Iandolo, B.; Nugroho, F. A. A.; Langhammer, C.; Lidström, E.; Wadell, C. Hysteresis-Free Nanoplasmonic Pd-Au Alloy Hydrogen Sensors. Nano Lett. 2015, 15, 3563-3570. 